\section{EL MUELLE DEL CENTENARIO EN EL PUERTO DE LA CORUÑA/ESPAÑA}

Ministerio de Obras Públicas y Urbanismo Dirección General de Puertos y Costas Junta del Puerto de La Coruña

$568-21$

\section{SINOPSIS}

Se describe en este artículo la ejecución de la obra del Muelle del Centenario, realizada en el Puerto de La Coruña.

Consiste en la construcción de cinco plataformas de hormigón armado sobre pilotes verticales in situ de $1,8 \mathrm{~m}$ de diámetro las filas de mar y tierra y $1,5 \mathrm{~m}$ las intermedias.

La longitud útil para el atraque de buques es de $540 \mathrm{~m}$ y el ancho transversal de $175 \mathrm{~m}$.

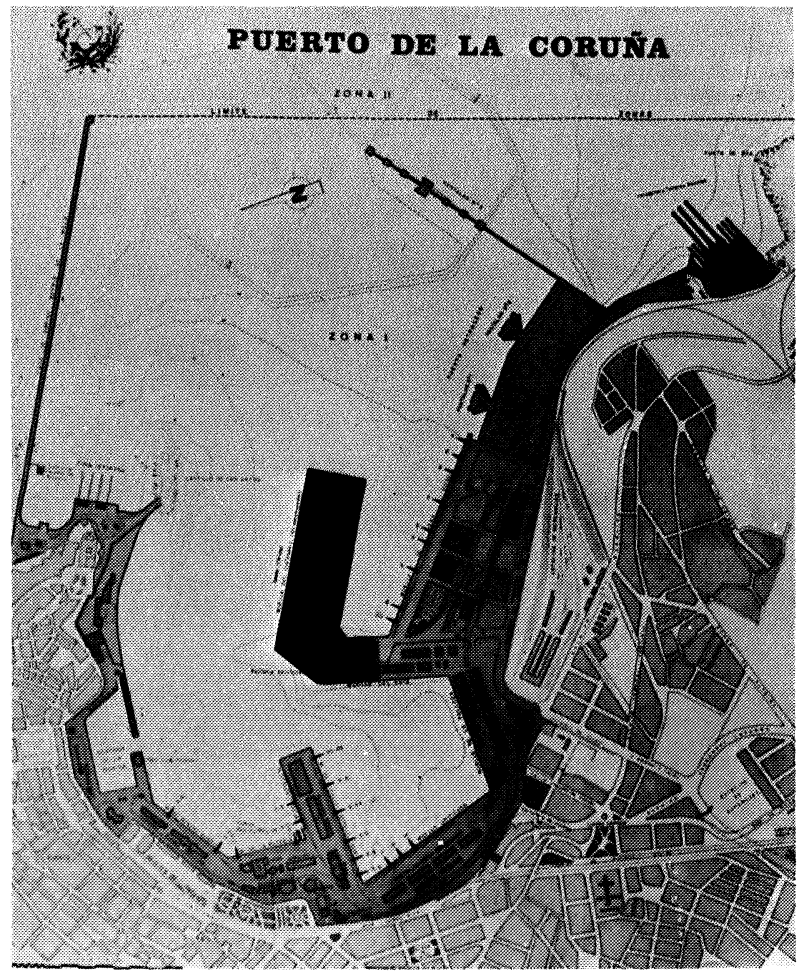

Coincidiendo con la conmemoración, en 1977, del primer centenario de la creación de la JUNTA DEL PUERTO DE LA CORUÑA, este Organismo decidio acometer la construcción de un nuevo muelle que permitiese a medio plazo satisfacer las necesidades previsibles de acuerdo con las tendencias observadas en las estadísticas del tráfico marítimo.

Efectivamente, si exceptuamos el petróleo, el resto de la carga seca tenía en ese momento una tasa de crecimiento acumulativo anual del $7 \%$. Con lo que de mantenerse dicha tendencia, el tráfico de 1985 duplicaría las cifras alcanzadas en 1976. Pero la realidad supero en mucho aquellas previsiones y en 1980 ya se doblaron los volúmenes de tráfico de 1976, creando graves problemas de congestión en las instalaciones existentes. Si añadimos a esto el papel preponderante que ha de ocupar el carbón en la atención de las necesidades energéticas españolas $y$, concretamente, en el entorno de influencia del Puerto de La Coruña, donde la demanda a medio plazo de carbón importado se prevé superior a los 2.000 .000 de $t$, resulta clara la conveniencia de disponer de un muelle polivalente, que pueda recibir el máximo de mercancias de cualquier tipo, y con una gran capacidad de almacenamiento y manipulación.

Un segundo factor determinante de la concepción del MUELLE DEL CENTENARIO fue la evolución seguida por el propio buque.

La tendencia actual en el transporte de graneles sólidos, y muy especialmente del carbón es que los buques alcancen entre las 120.000 y 150.000 toneladas peso muerto. Este tipo de transporte exige la creación de plataformas o muelles de gran capacidad de almacenamiento y con calado suficiente.

Finalmente, se creó el emplazamiento óptimo dentro de la actual configuración del Puerto de la Coruña que venía dictado por la doble necesidad de una buena accesibilidad terrestre y maritima, sin entorpecer la utilización de los muelles existentes. Esto hizo que el futuro MUELLE DEL CENTENARIO se situará en una zona cuyos fondos están constituidos por una capa de fangos de unos 14 metros de espesor y con nulas caracteristicas resistentes en su estado actual.

En base a estas consideraciones, el 4 de enero de 1978, la JUNTA DEL PUERTO DE LA CORUÑA convocb un Concurso para la redacción del Anteproyecto del NUEVO MUELLE DE SAN DIEGOMUELLE DEL CENTENARIO y posterior realización de las obras correspondientes al Proyecto de acuerdo con la oferta seleccionada. 

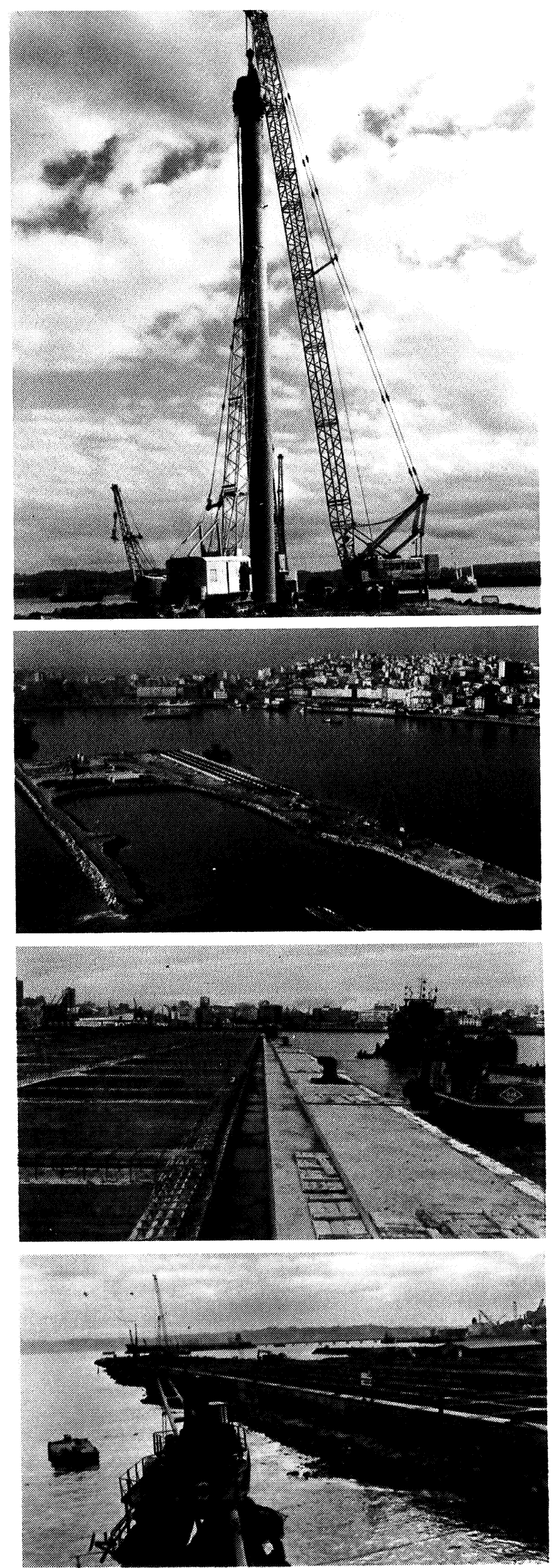

De entre los Anteproyectos concursantes, la JUNTA DEL PUERTO DE LA CORUÑA selecciono la solución que se describe a continuación:

Consiste en la construcción de nueve plataformas de hormigón armado, con un total de $540 \mathrm{~m}$ de Iongitud atracable, que encepan cuatro filas de pilotes verticales in situ de hormigón armado, con diámetro de $1,80 \mathrm{~m}$ las filas de mar y tierra, y de $1,50 \mathrm{~m}$ las filas intermedias. El número total de pilotes es de 280

Para evitar las complicaciones inherentes a la ejecución a flote de un pilotaje de gran diámetro, se opto por realizarlo en seco, mediante el vertido previo de tierras a lo largo de las futuras plataformas, preconsolidando asi hasta un $75 \%$ los fangos subyacentes. Una vez ejecutado el pilotaje, cuyas longitudes varian entre 25 y $40 \mathrm{~m}$, se draga dicho vertido, de forma que los rellenos del terraplén del muelle extiendan su talud definitivo, con su correspondiente capa protectora de escollera, por debajo de las plataformas, en cuyos pilotes, y dada la preconsolidación alcanzada, el rozamiento negativo y los esfuerzos transversales entran dentro de los límites admisibles. El ancho transversal del muelle - plataformas más terraplenes - alcanza asi los 175 metros.

La anchura de las plataformas es de $28,95 \mathrm{~m}$ con vigas de $2,05 \mathrm{~m}$ de canto. La viga cantil tiene un ancho de 3,85 $\mathrm{m}$ y es aligerada, alojando una galería de servicios de $1,10 \times 1,20 \mathrm{~m}$. Esta viga lleva un faldón hasta la cota $+2,00$. Las vigas intermedias tienen $90 \mathrm{~cm}$ de ancho y son macizas as como la viga de tierra que tiene una anchura de $2 \mathrm{~m}$.

La losa superior de las plataformas es de $40 \mathrm{~cm}$ de espesor con terminación a la cota $+6,00$.

Posteriormente se efectúa un dragado a la cota -14 en toda la zona frontal al nuevo muelle, de anchura variable entre $150 \mathrm{~m}$ y $225 \mathrm{~m}$, profundizándose hasta la $-16,50$ en una fosa adyacente al cantil, de $60 \mathrm{~m}$ de anchura.

Finalmente se disponen bolardos y defensas para buques de $150.000 \mathrm{tpm}$, así como vías de rodadura para grúas pórtico con una capacidad para $50 \mathrm{t}$, alcance máximo de $35 \mathrm{~m}$ y ancho de vía de 15,75 metros; y grúas pórtico de $16 \mathrm{t}$, alcance máximo de $35 \mathrm{~m}$ y ancho de vía de $9 \mathrm{~m}$, y tres vías tipo RENFE con sus correspondientes enlaces.

Los ordenes de magnitud de las obras pueden estimarse por los siguientes volúmenes globales:

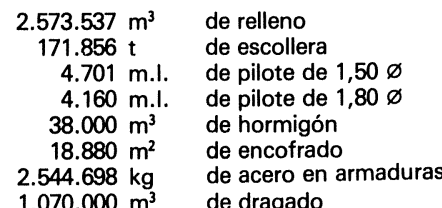

Empresa

Constructora: ENTRECANALES Y TAVORA, S. A. 